\title{
A NUMERICAL STUDY ON LONG-WAVE GENERATION DUE TO ATMOSPHERIC-PRESSURE VARIATION
}

\author{
Taro Kakinuma ${ }^{1}$ and Kousuke Fukita ${ }^{2}$
}

\begin{abstract}
The generation and propagation of long waves due to atmospheric-pressure variation have been numerically simulated, where the atmospheric-pressure waves are assumed to travel in the eastern direction over East Chine Sea. The wave height of long waves is larger as the moving velocity of the recovery point of water surface pressed by the atmospheric pressure is close to the long-wave celerity. Before attenuation of the harbor oscillation in Urauchi Bay, Kamikoshiki Island, the incidence of long waves can continue because of the oscillation system generated between the main island of Kyushu and Okinawa Trough. The pressure profiles of atmospheric-pressure waves supposed to have concerned the large harbor oscillation in Urauchi Bay from 2009 to 2010 are classified into four patterns based on the Grid Point Value pressure data. The incident long waves causing the largest harbor oscillation in Urauchi Bay on Feb. 25, 2009 has been simulated with the estimated pressure-profile pattern, such that the largest harbor oscillation in Urauchi Bay should occur due to the atmospheric-pressure waves.
\end{abstract}

Keywords: long wave; atmospheric pressure; harbor oscillation; seiche; East China Sea

\section{INTRODUCTION}

During Feb. 24-26, 2009, eight fishing boats capsized and several houses were flooded due to heavy harbor oscillation, where the total amplitude of water level reached three meters, in Urauchi Bay of Kamikoshiki Island situated in the western offing of Kyushu Island, Japan (Kakinuma et al., 2009a; Kakinuma et al., 2009b). In the present study, such harbor oscillation in the south of Kyushu is assumed to be caused by incidence of long waves due to atmospheric-pressure variation over the East Chine Sea (Hibiya and Kajiura, 1982) and long-wave generation is numerically simulated when atmosphericpressure waves propagate toward the east. The long waves causing the largest harbor oscillation in Urauchi Bay on Feb. 25, 2009 is also reproduced with an estimated pressure-profile pattern of atmospheric-pressure waves.

\section{NUMERICAL MODEL AND CALCULATION CONDITIONS}

A set of nonlinear shallow water equations in consideration of atmospheric-pressure gradient is solved in the horizontal two-dimension by applying a finite difference method. The fundamental equations are

$$
\begin{gathered}
\frac{\partial \eta}{\partial t}+\frac{\partial}{\partial x}\{(\eta+h) U\}+\frac{\partial}{\partial y}\{(\eta+h) V\}=0 \\
\frac{\partial U}{\partial t}+\frac{\partial U^{2}}{\partial x}+\frac{\partial(U V)}{\partial y}=f V-g \frac{\partial \eta}{\partial x}-\frac{1}{\rho} \frac{\partial P}{\partial x}+A_{h}\left(\frac{\partial^{2} U}{\partial x^{2}}+\frac{\partial^{2} U}{\partial y^{2}}\right)-\frac{K U \sqrt{U^{2}+V^{2}}}{\eta+h} \\
\frac{\partial V}{\partial t}+\frac{\partial(U V)}{\partial x}+\frac{\partial V^{2}}{\partial y}=-f U-g \frac{\partial \eta}{\partial y}-\frac{1}{\rho} \frac{\partial P}{\partial y}+A_{h}\left(\frac{\partial^{2} V}{\partial x^{2}}+\frac{\partial^{2} V}{\partial y^{2}}\right)-\frac{K V \sqrt{U^{2}+V^{2}}}{\eta+h}
\end{gathered}
$$

where $U$ and $V$ are horizontal velocities in the $x$ and $y$ directions, respectively; $\eta, h$, and $P$ are water surface displacement, still water depth, and atmospheric pressure on water surface, respectively. In the present study, Coriolis coefficient $f=7.3 \times 10^{-5} \mathrm{~s}^{-1}$, gravitational acceleration $g=9.8 \mathrm{~m} / \mathrm{s}^{2}$, seabed friction coefficient $K=2.6 \times 10^{-3}$, horizontal eddy viscosity coefficient $A_{h}=100.0 \mathrm{~m}^{2} / \mathrm{s}$, and sea water density $\rho=1035.0 \mathrm{~kg} / \mathrm{m}^{3}$. The radiation condition is adopted on boundaries in water areas, while boundaries between land and sea are assumed to be perfect-reflection walls.

The atmospheric-pressure waves are classified into four patterns as shown in Fig. 1 based on the Grid Point Value (GPV) pressure data, which are published by Japan Meteorological Agency, to examine the relationship between the atmospheric-pressure waves and long-wave generation over the

\footnotetext{
${ }^{1}$ Division of Natural Science, Graduate School of Science and Engineering, Kagoshima University, 1-21-40 Korimoto, Kagoshima, Kagoshima 890-0065, Japan

${ }^{2}$ West Japan Engineering Consultants, Inc., 1-1-1 Watanabe-dori, Chuo-ku, Fukuoka 810-0004, Japan
} 


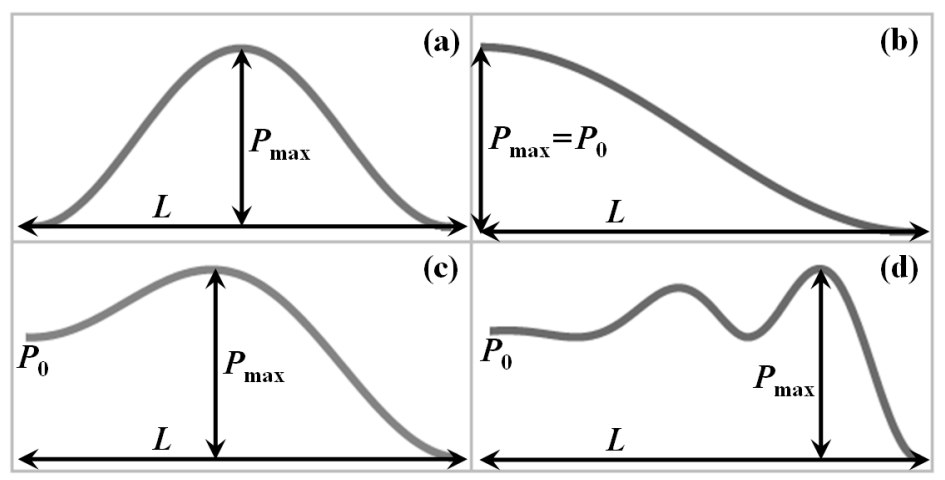

Figure 1. Pressure profiles of atmospheric-pressure waves.

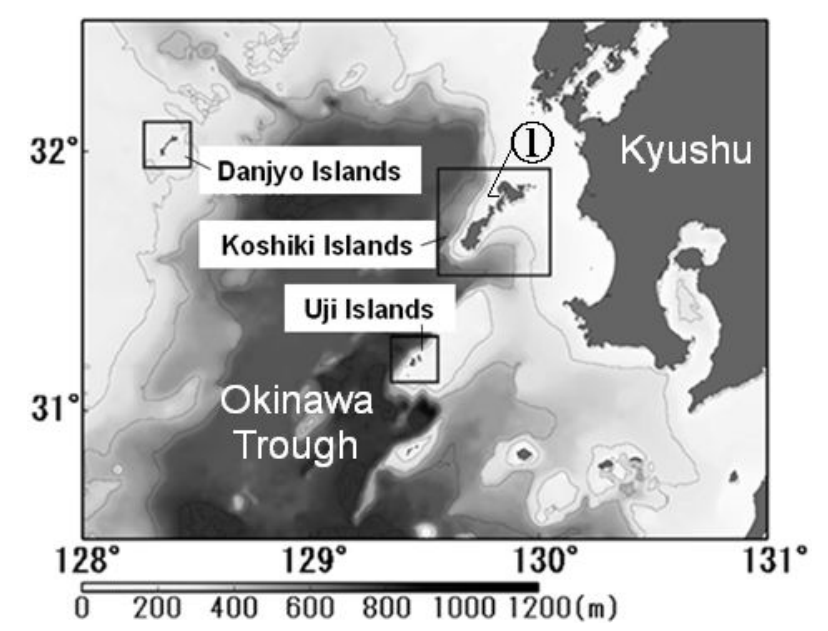

Figure 2. Still water depth in a part of the calculation domain.

actual seabed configuration within $123-131^{\circ} \mathrm{E}$ and $30-32.5^{\circ} \mathrm{N}$ of East China Sea. Pattern (a), for example, has three parameters, i.e., total wavelength $L$, maximum value of pressure, $P_{\max }$, and moving velocity $C_{p}$.

The still water depth in a part of the calculation domain is shown in Fig. 2. Over East China Sea, the atmospheric pressure is assumed to be uniform from north to south, travelling to the east.

In numerical computation, the grid widths and the time-step interval are equal to $\Delta x=790 \mathrm{~m}, \Delta y=$ $925 \mathrm{~m}$, and $\Delta t=2 \mathrm{~s}$, respectively.

\section{NUMERICAL RESULTS}

\section{Relationship between the Parameters of Atmospheric-Pressure Waves and Long-Wave \\ Generation}

In Pattern (a) of Fig. 1, the pressure profile of atmospheric-pressure wave is described as

$$
P\left(x, t_{0}\right)= \begin{cases}\frac{p_{\max }}{2}\left\{1+\cos \left[\frac{2 \pi}{L}\left(x-x_{c}\right)\right]\right\} & \left(\left|x-x_{c}\right| \leq L / 2\right), \\ 0 & \left(\left|x-x_{c}\right|>L / 2\right)\end{cases}
$$

where the initial position of the pressure peak, $x_{c}$, is $124^{\circ} \mathrm{E}$.

Figure 3 shows the water surface displacement at Point (1) in Fig. 2 due to the assumed atmospheric-pressure waves of Pattern (a), where $L=10 \mathrm{~km}, C_{p}=20 \mathrm{~m} / \mathrm{s}$, and $P_{\max }=1$, 2, or $3 \mathrm{hPa}$. The still water depth is about $22.0 \mathrm{~m}$ at Point (1). The wave height of generated long waves is almost in proportion to $P_{\max }$, which has been confirmed at the other monitoring points.

Figure 4 shows the wave height and wave period of long waves of the maximum wave height at 


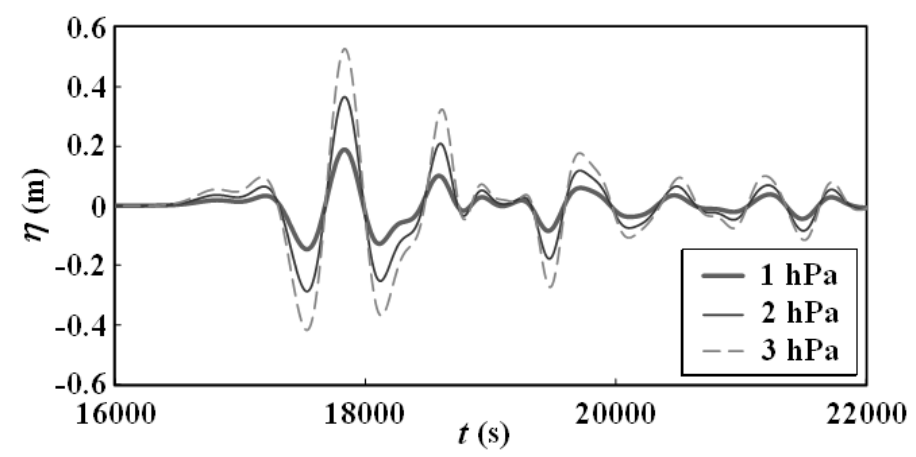

Figure 3. Water surface displacement at Point (1) (The pressure-profile pattern is Pattern (a), where $L=10 \mathrm{~km}$, $C_{p}=20 \mathrm{~m} / \mathrm{s}$, and $P_{\max }=1,2$, or $3 \mathrm{hPa}$.).

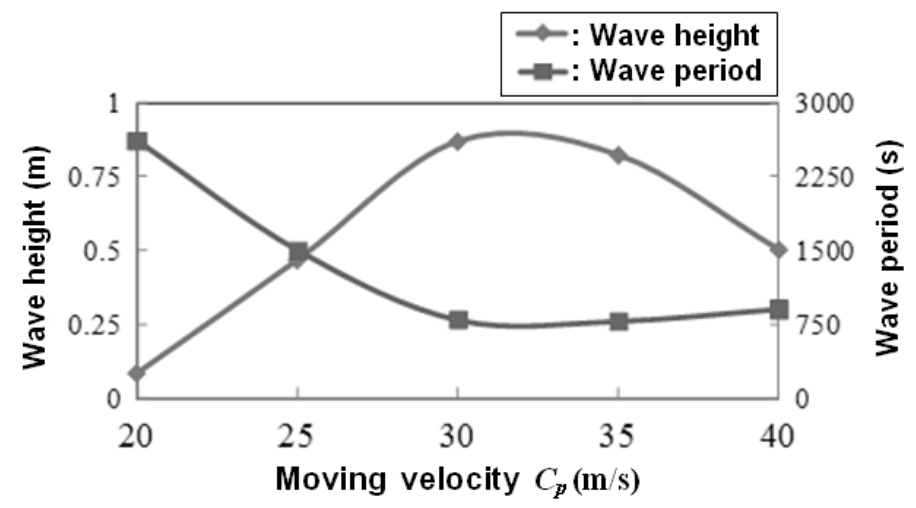

Figure 4. Wave height and wave period of long waves of the maximum wave height at Point (1) (The pressure-profile pattern is Pattern (a), where $L=30 \mathrm{~km}$ and $P_{\max }=1 \mathrm{hPa}$.).

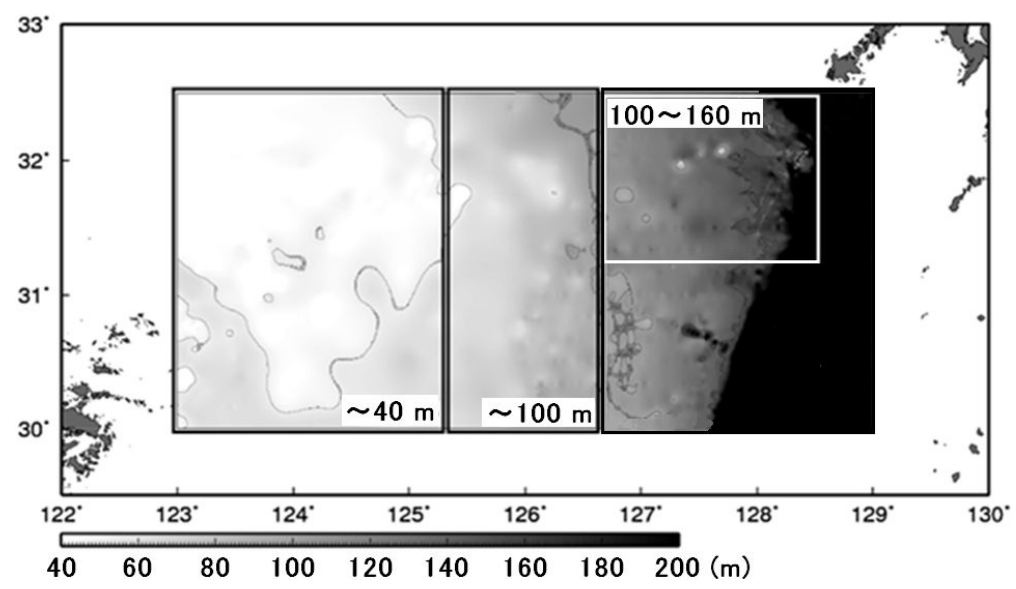

Figure 5. Average water depth in East China Sea.

Point (1) with Pattern (a), where $L=30 \mathrm{~km}$ and $P_{\max }=1 \mathrm{hPa}$. The waves are defined through the zeroup-cross method. The wave height becomes large when $C_{p}$ is around $32 \mathrm{~m} / \mathrm{s}$. Hibiya and Kajiura (1982) concluded that the large harbor oscillation in Nagasaki Bay in the north of Kyushu was caused by large incident long waves due to a resonance in East China Sea. According to Fig. 5, where the average water depth is shown inside each frame, the average water depth over the continental shelf is $80 \mathrm{~m}$ or $100 \mathrm{~m}$, such that the long-wave celerity is around $30 \mathrm{~m} / \mathrm{s}$, resulting in the amplification of wave height caused by the resonance between the atmospheric-pressure wave and long waves. 


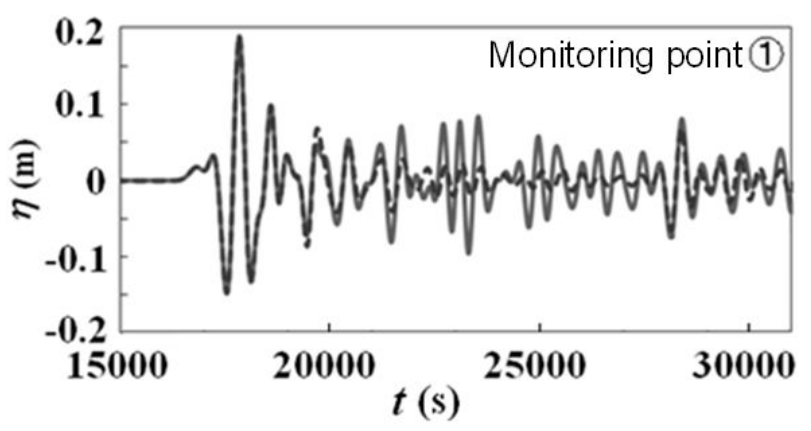

Figure 6. Water surface displacements at Point (1) (The pressure-profile pattern is Pattern (a), where $L=10$ $\mathrm{km}, P_{\max }=1 \mathrm{hPa}$, and $C_{\mathrm{p}}=20 \mathrm{~m} / \mathrm{s}$.; The solid and broken lines show the results with and without existence of the main island of Kyushu, respectively.).

\section{Oscillation System between the Main Island of Kyushu and Okinawa Trough}

Large harbor oscillation requires continuous incidence of wave energy into Urauchi Bay (Kakinuma et al., 2009a). Figure 6 shows the water surface displacements at Point (1) off Urauch Bay using Pattern (a), where $L=10 \mathrm{~km}, P_{\max }=1 \mathrm{hPa}$, and $C_{p}=20 \mathrm{~m} / \mathrm{s}$. In the figure, the numerical result including wave reflection at the west edge of the main island of Kyushu is compared with that without existence of the main island of Kyushu. In the latter case, the target domain of numerical calculation is only the area within $123-130^{\circ} \mathrm{E}$. An oscillation system is generated off the south of Kyushu between the main island of Kyushu and Okinawa Trough, resulting in the continuing motion of water surface to make larger harbor oscillation in Urauchi Bay, whereas such oscillation off the north of Kyushu may appear among the main and other islands.

\section{Long Waves on the Days when Large Harbor Oscillation Occurred in Urauchi Bay}

In Patterns (b), (c), and (d) shown in Fig. 1, the pressure profiles of atmospheric-pressure waves are written as

$$
\begin{aligned}
\text { (b) : } P\left(x, t_{0}\right)= & P_{0}\left\{1-\sin \left[\pi\left(x-x_{c}\right) / L\right]\right\} / 2, \\
\text { (c) : } P\left(x, t_{0}\right)= & P_{0}\left\{1+\cos \left[2 \pi\left(x-x_{c}\right) / L\right]\right\} / 2 \\
& +P_{0}\left\{1-\sin \left[\pi\left(x-x_{c}\right) / L\right]\right\} / 2, \\
\text { (d) }: P\left(x, t_{0}\right)= & 0.05 e^{\kappa} P_{0}\left\{1+\cos \left[6 \pi\left(x-x_{c}\right) / L\right]\right\} / 4 \\
& +P_{0}\left\{1-\sin \left[3 \pi\left(x-x_{c}-x_{d}\right) / L\right]\right\} / 2,
\end{aligned}
$$

respectively, where $\kappa=0.02 x$ and $x_{c}$ is the initial position of the second peak of Pattern (d), when $\left|x-x_{c}\right| \leq L / 2 ; P\left(x, t_{0}\right)=P_{0}\left(x<x_{c}-L / 2\right)$ and $P\left(x, t_{0}\right)=0\left(x>x_{c}+L / 2\right)$.

The parameters of each pattern are evaluated according to the GPV pressure data on the days of large harbor oscillation in Urauchi Bay. For example, the time variation of the GPV pressure on Feb. 25, 2009, i.e., the day with the largest harbor oscillation in Urauchi Bay, is shown in Fig. 7 and the pressure distributions along latitude lines of the figure on 3:00 (JST) in Fig. 7 are shown in Fig. 8. These figures indicate that an atmospheric-pressure wave, whose pressure gap was 4 or $5 \mathrm{hPa}$ and total wavelength was 80 or $120 \mathrm{~km}$, traveled almost towards the east over East China Sea; its moving velocity was around $140 \mathrm{~km} / \mathrm{h}(3: 00-4: 00), 120 \mathrm{~km} / \mathrm{h}(4: 00-5: 00)$, and $150 \mathrm{~km} / \mathrm{h}(5: 00-6: 00)$.

The days when especially large harbor oscillation occurred in Urauchi Bay from 2009 to 2010 were Feb. 25, 2009, Mar. 3, 2010, Mar. 5, 2010, and Mar. 6, 2010 (Department of Ocean Civil Engineering, Kagoshima University, 2010), such that the GPV pressure data on these four days are used to evaluate the parameters of pressure profiles of atmospheric-pressure waves. Consequently the mean values of $(L$, $\left.P_{\max }, C_{p}\right)$ are equal to $(100.0 \mathrm{~km}, 3.0 \mathrm{hPa}, 20.0 \mathrm{~m} / \mathrm{s}),(100.0 \mathrm{~km}, 4.0 \mathrm{hPa}, 33.0 \mathrm{~m} / \mathrm{s})$, and $(90.0 \mathrm{~km}, 4.0$ $\mathrm{hPa}, 25.0 \mathrm{~m} / \mathrm{s}$ ) in Patterns (b), (c), and (d), respectively.

Numerical calculation results of water surface displacements at Point (1) are shown in Fig. 9 for the assumed atmospheric-pressure waves of Patterns (b), (c), and (d). Long waves are not generated 

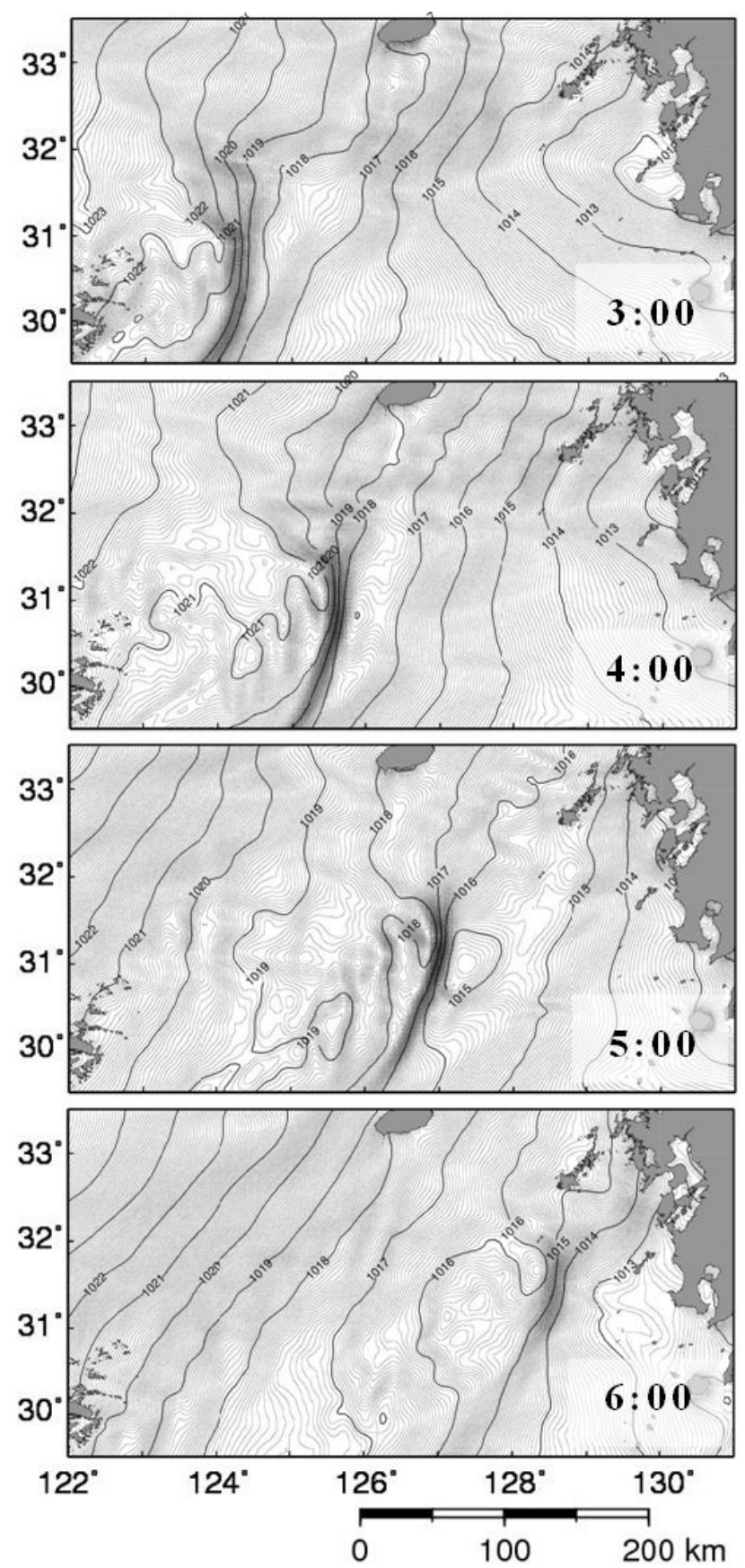

Figure 7. Time variation of the GPV pressure on Feb. 25, 2009 (time: Japan Standard Time).

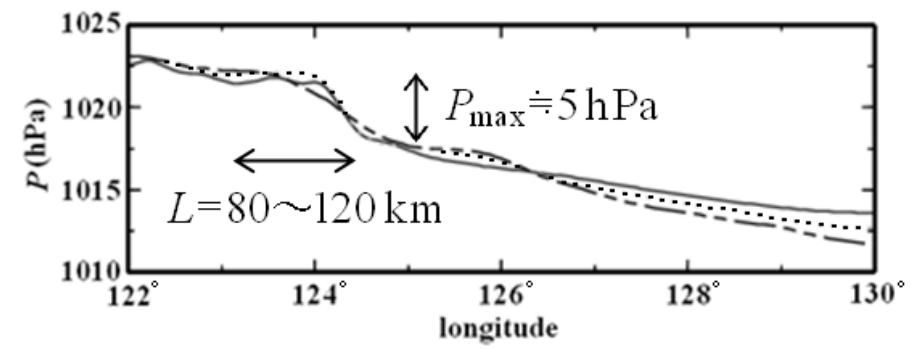

Figure 8. Pressure distributions along the latitude lines of the figure on 3:00 in Fig. 7 (The solid, dotted, and chain double-dashed lines show the pressure at the latitude of $30^{\circ} \mathrm{N}, 30.5^{\circ} \mathrm{N}$, and $31^{\circ} \mathrm{N}$, respectively.) 


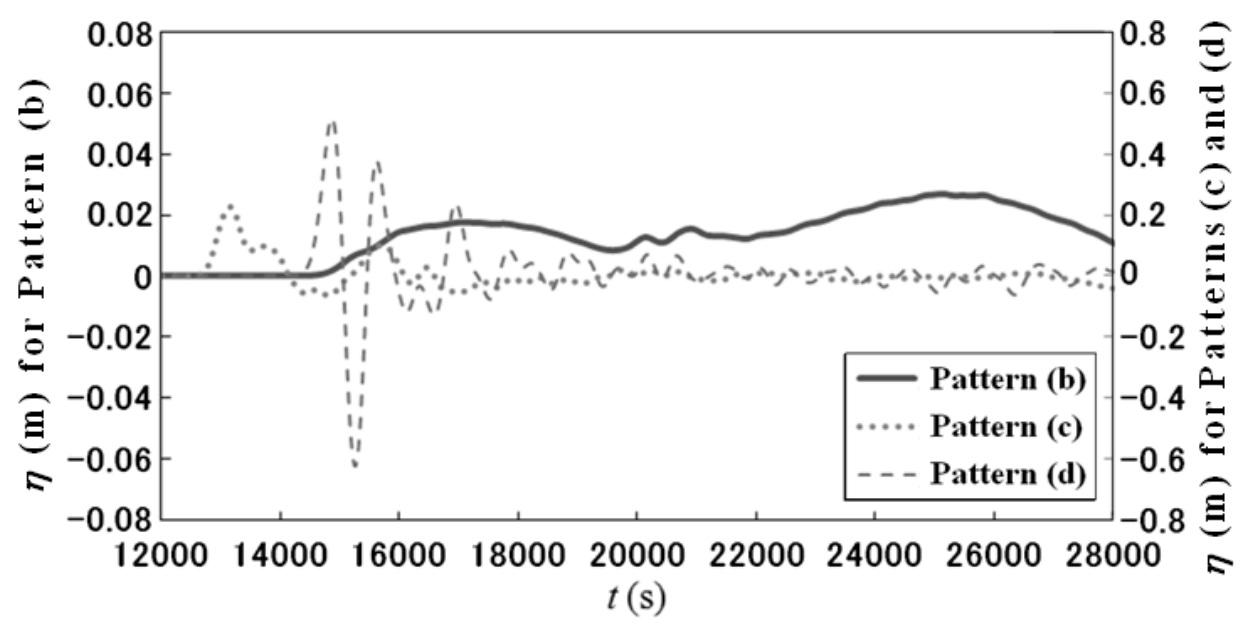

Figure 9. Water surface displacements at Point (1).

remarkably due to the atmospheric-pressure wave of Pattern (b) because this pattern has no pressure drop after the pressure-rise process, i.e., the sea surface, which has been pressed down, is not relieved to become free-surface waves. The resonance described above, i.e., the resonance between an atmospheric-pressure wave and a long sea wave, appears when the moving velocity of a recovery point of water surface pressed by the atmospheric pressure is close to the long-wave celerity. On the other hand, the long waves due to the atmospheric-pressure wave of Pattern (c) obtain the maximum wave height of about $0.3 \mathrm{~m}$, where the wave of the maximum wave height has wave period of around 2,600 s; the long waves for Pattern (d) show that the wave height and wave period of the first wave are about 1.1 $\mathrm{m}$ and 1,300 s, respectively, while the wave period of the second and the third waves are 1,250 $\mathrm{s}$ and $900 \mathrm{~s}$, respectively.

\section{Long Waves Causing the Largest Harbor Oscillation in Urauchi Bay}

The largest harbor oscillation was observed in Urauchi Bay on Feb. 25, 2009 and the pressure profiles of atmospheric-pressure waves are categorized as Patten (d), where $L=90.0 \mathrm{~km}, P_{\max }=4.0 \mathrm{hPa}$, and $C_{p}=38.6 \mathrm{~m} / \mathrm{s}$ according to the GPV pressure data. Numerical computation results of time variation of water-level distribution are shown in Fig. 10, where the waves show refraction over Okinawa Trough, after which they propagate to the northeast.

The calculated water surface displacement at Point (1) is shown in Fig. 11, where the wave period is about 1,000, 750, 700, 760, and $660 \mathrm{~s}$ from the first to the fifth waves, respectively, and the wave height of the first three waves is over one meter. Kakinuma et al. (2009b) performed numerical simulation of harbor oscillation in Urauchi Bay and mentioned that harbor oscillation of total amplitude up to $3 \mathrm{~m}$ could appear in Urauchi Bay due to more than two incident long waves whose wave height and wave period were around $0.54 \mathrm{~m}$ and $720 \mathrm{~s}$, respectively, such that the largest oscillation in Urauchi Bay on Feb. 25, 2009 should occur due to the atmospheric-pressure waves of the evaluated pressure-profile pattern.

\section{CONCLUSIONS}

The generation and propagation of long waves due to atmospheric-pressure waves have been numerically simulated using the set of nonlinear shallow water equations, where the atmosphericpressure waves are assumed to travel in the eastern direction over East Chine Sea. The wave height of long waves is larger as the moving velocity of the recovery point of water surface pressed by the atmospheric pressure is close to the long-wave celerity. Before attenuation of the harbor oscillation in Urauchi Bay, the incidence of long waves can continue because of the oscillation system generated between the main island of Kyushu and Okinawa Trough.

The pressure profiles of atmospheric-pressure waves are classified into four patterns and the parameters of three of them are evaluated according to the GPV pressure data on four days with especially large harbor oscillation in Urauchi Bay. The incident long waves causing the largest harbor oscillation in Urauchi Bay on Feb. 25, 2009 has been simulated with the evaluated pressure-profile 


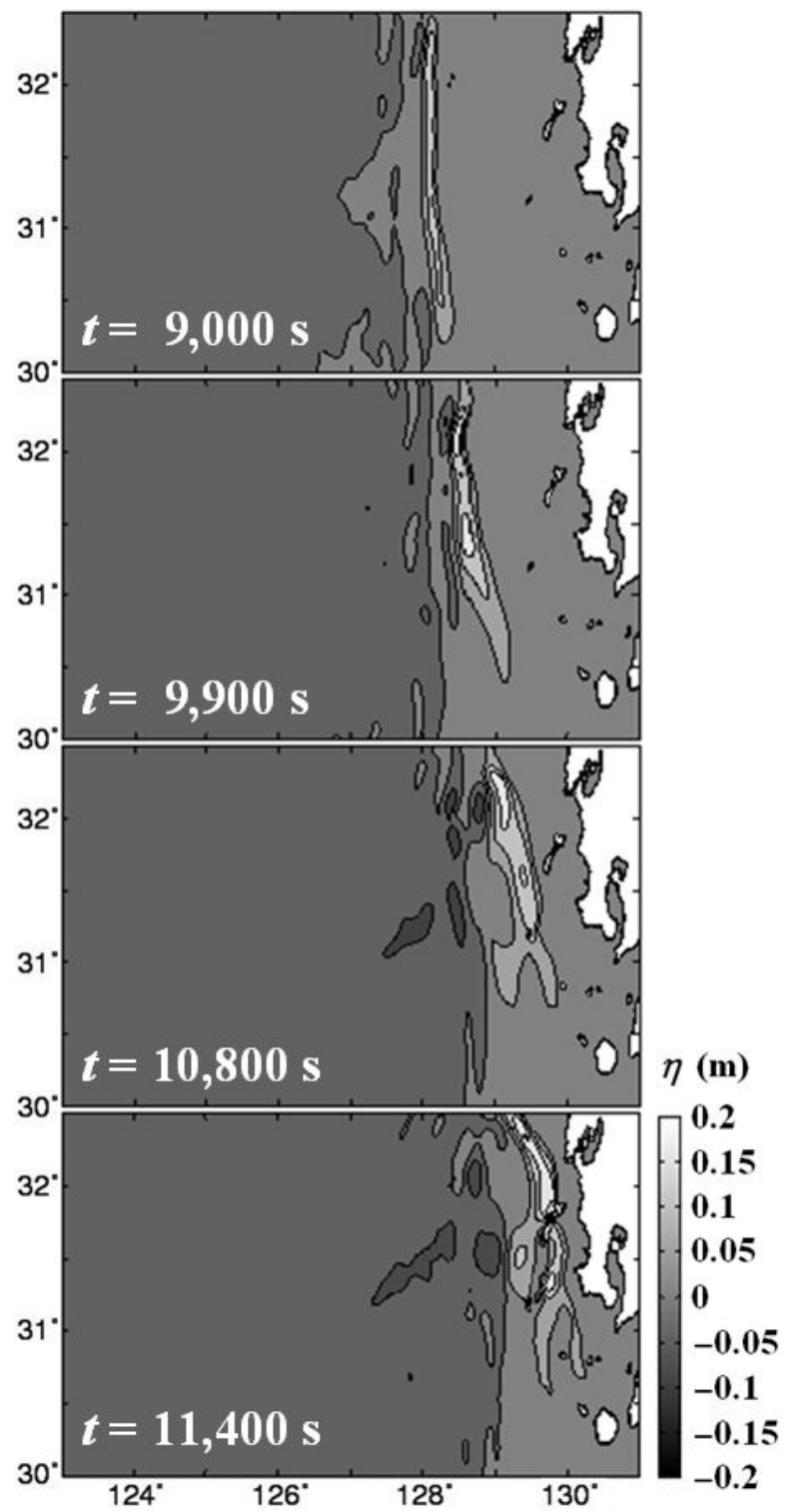

Figure 10. Time variation of water-level distribution on Feb. 25, 2009.

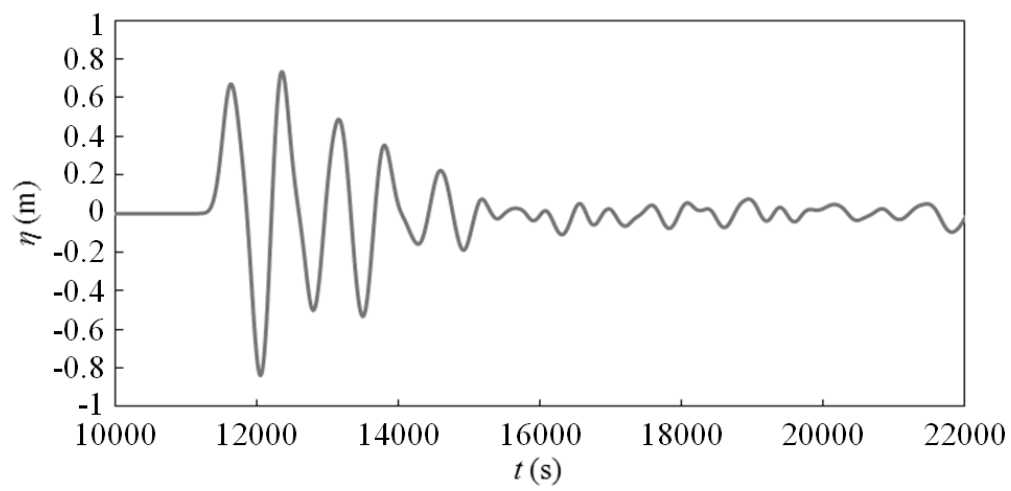

Figure 11. Water surface displacement at Point (1) on Feb. 25, 2009.

pattern, such that the largest harbor oscillation in Urauchi Bay should occur due to the atmosphericpressure waves. 


\section{REFERENCES}

Department of Ocean Civil Engineering, Kagoshima University. 2010. Report on Countermeasures against Harbor Oscillation in the Area around Koshiki Islands, Department of Ocean Civil Engineering, Kagoshima University, 226p. (in Japanese).

Hibiya, T . and K. Kajiura. 1982. Origin of the Abiki phenomenon (a kind of seiche) in Nagasaki Bay, Journal of Oceanography, 38, 172-182.

Kakinuma, T., T. Toyofuku, and T. Inoue. 2009a. Numerical analysis of harbor oscillation in harbors of various shapes, Proceedings of Coastal Dynamics 2009, World Scientific Publishing Co.

Kakinuma, T., T. Asano, T. Inoue, T. Yamashiro, and K. Yasuda. 2009b. Survey on February 2009 Abiki Disaster in Urauchi Bay, Kamikoshiki Island, Journal of Japan Society of Civil Engineers, B2-65, 1, 1391-1395 (in Japanese). 